\title{
control continuo de uniformidad en los alambres de acero para hormigón pretensado
}

\section{J. M. TOBIO, Dr. en Química Industrial}

464.1

\section{Ceneralidades}

La mayor parte de los hilos o alambres utilizados en el hormigón pretensado proceden de partidas de acero Martín, que, al parecer, son las de composición más regular y las más exentas de inclusiones de nitrógeno (1). También los aceros procedentes de horno eléctrico resultan apropiados para este tipo de alambres (2).

Los aceros normales para pretensado, usuales en la actualidad, poseen la composición química aproximada siguiente:

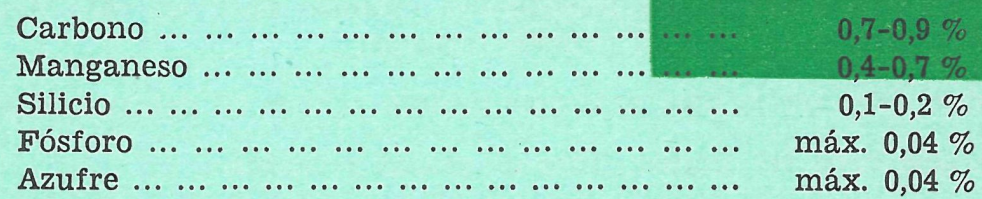

En los aceros tratados térmicamente se admiten porcentajes algo más altos en manganeso $(0,7-1,5 \%)$ y en silicio $(0,8-1,2 \%)$.

Fundamentalmente, los alambres de acero para estructuras pretensadas pueden dividirse en los dos grandes grupos siguientes:

1. Alambres que adquieren sus propiedades mecánicas por tratamiento en frío.

2. Alambres que adquieren sus propiedades mecánicas por tratamiento térmico.

Los principales tratamientos seguidos en la obtención de alambres para pretensado pueden resumirse así:

Templado por inmersión (Patenting).-Consiste en calentar el alambre a $800-900^{\circ} \mathrm{C} \mathrm{y}$ luego enfriarlo en un segundo a unos $500^{\circ} \mathrm{C}$ por inmersión en plomo fundido, en el cual se tiene durante algún tiempo a esta temperatura. Después se deja enfriar a la temperatura ambiente. Se obtiene así una estructura sorbítica, que se presta muy bien para un estirado en frío posterior.

Estirado en frío.-Se hace disminuir el diámetro del alambre pasándolo a través de una hilera. El acero adquiere una textura alargada. La temperatura alcanzada durante el estirado es de $100-150^{\circ} \mathrm{C}$.

Los alambres templados por inmersión y luego estirados en frío pueden alcanzar resistencias a tracción de hasta $300 \mathrm{~kg} / \mathrm{mm}^{2}$.

Laminado en frío. Se lleva a cabo pasando el alambre por rodillos o cilindros. La diferencia con el estirado en frío es simplemente la ausencia de fricción sobre la superficie del alambre durante el laminado.

Templado.-Se verifica enfriando el alambre bruscamente desde los $800-900^{\circ} \mathrm{C}$ hasta la temperatura ambiente. La estructura del acero es entonces martensítica, en forma de agujas. Este acero es muy duro, pero quebradizo. 
Semitemple.-Para evitar tensiones térmicas en el acero, debidas al proceso de endurecimiento, es posible enfriarlo bruscamente de $800-900^{\circ} \mathrm{C}$ a $300^{\circ} \mathrm{C}$. Se mantiene así durante algún tiempo y luego se enfría a la temperatura ambiente. Este alambre no se suele someter al estirado en frío.

Destensionado.-Es un tratamiento térmico al que suele someterse el acero templado. Se mantiene el alambre durante algún tiempo a $200-250^{\circ} \mathrm{C}$, durante cuyo período hay una segregación en la malla cristalina y una eliminación de tensiones en el acero.

Revenido.--También se hace con alambre templado. Se verifica a $400-500^{\circ} \mathrm{C}$, y las tensiones internas del acero, debidas al proceso de temple, se neutralizan, cubriéndose la estructura acicular martensítica con una estructura intermedia más blanda.

Existen, además, en el proceso de fabricación, otras operaciones tales como el calibrado, torsionado, tratamientos isotérmicos en baños salinos y otras. Posiblemente algunos de los tratamientos antes descritos se aplican sucesivamente para dar los alambres de acero de alta resistencia.

Para que el proceso de fabricación pueda llevarse a cabo con toda garantía, es necesario partir de primeras materias (acero) de calidad y libres de inclusiones. Los tratamientos térmicos deben realizarse cuidadosamente, y si se verifican soldaduras (para el estirado en frío, por ejemplo) deben marcarse bien en el alambre para eliminar luego esas zonas.

En todo caso, el fabricante debe preocuparse de obtener alambres totalmente homogéneos, y no crear, durante los tratamientos, tensiones innecesarias en el acero. Pero, aun con todo, es muy difícil obtener alambres que no presenten alguna imperfección.

\section{Propiedades que deben reunir los aceros}

Es muy difícil resumir en pocas líneas las propiedades que debe poseer un alambre de acero para pretensado. Además, las propiedades óptimas para un caso determinado pueden no serlo para otro. De un modo general diremos:

a) El alambre debe ser lo más uniforme posible y libre de defectos visibles.

b) Su superficie debe ser limpia, libre de grasas y óxidos.

c) El diámetro especificado para los alambres debe mantenerse entre $+4 \%$ y $-2 \%$ para los estirados en frío $\mathrm{y}+10 \% \mathrm{y}-2 \%$ para los laminados.

d) El alambre, dentro de cada rollo, debe ser continuo, sin soldadura de ningún género.

e) Debe ser recto. El diámetro de los rollos ha de ser tal que, cuando se desenvuelva el hilo, no debe desviarse de la línea recta más de $10 \mathrm{~cm}$ por metro.

f) Los rollos deben venir amarrados, al menos con seis fuertes alambres.

g) El peso de los rollos debe ser:

Hasta $25 \mathrm{~mm}^{2}$ de sección .......... $>50 \mathrm{~kg}$.

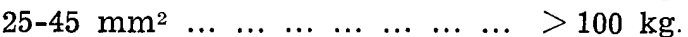

$>45 \mathrm{~mm}^{2} \ldots \ldots \ldots \ldots \ldots \ldots \ldots+150 \mathrm{~kg}$.

h) El alambre debe satisfacer los ensayos de tracción, carga de rotura, alargamiento, doblado, torsión, fluencia y cualquier otro marcado por las especificaciones correspondientes, dentro de los límites de tolerancia prescritos.

\section{Fallos en los aceros}

A veces se producen roturas espontáneas en los alambres, atribuibles a las siguientes causas:

a) Defectos mecánicos en los alambres o en las soldaduras de los mismos, si los trozos soldados no han sido cuidadosamente eliminados.

b) La resistencia a la fatiga del acero puede ser insuficiente para la carga utilizada.

c) Pueden existir tensiones internas resultantes del proceso de fabricación.

d) Errores en la colocación de los alambres: anclajes, entalladuras provocadas durante la colocación, dobleces, torsiones, etc. 
e) Los hilos pueden romperse por corrosión bajo tensión. Este fenómeno puede ocurrir con alguna frecuencia en los aceros templados y revenidos.

En términos generales, las roturas espontáneas se deben casi siempre a fenómenos químicos o electroquímicos, es decir, a la corrosión bajo tensión indicada en e) (3).

\section{Corrosión bajo tensión}

Prácticamente todos los aceros utilizados en pretensado son aceros al carbono o ligeramente aleados. No poseen, por tanto, ninguna resistencia a la corrosión y, por ello, deben ser convenientemente protegidos de este tipo de ataque. El hormigón, por otra parte, constituye un buen recubrimiento anticorrosivo.

Los alambres de aceros más duros son los que están más expuestos a la corrosión bajo tensión (1).

La corrosión normal es muy diferente de la corrosión bajo tensión. En aquélla, el acero se recubre de una capa de óxido (óxido-hidróxido-carbonato); el ataque hace disminuir la sección y el alambre puede romperse por esta causa.

En la corrosión bajo tensión, que es en realidad un ataque intercristalino, se trata de una forma de corrosión localizada, ocasionada por un ataque químico o electroquímico combinado con elevadas tensiones mecánicas y cargas, que conduce a la formación de fisuras capilares entre los cristales. Excepcionalmente, estas fisuras pueden producirse dentro de los propios cristales. Estas fisuras son puntos de iniciación de rotura.

Es algo así como si la malla cristalina del acero se "aflojase" por efecto de las tensiones, dando una fácil entrada a la corrosión.

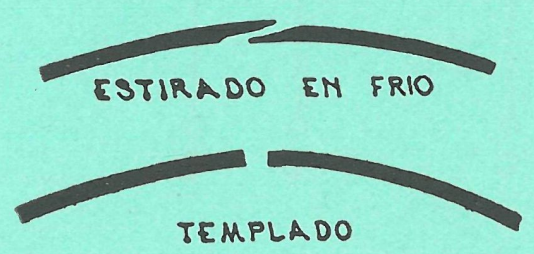

Las roturas pueden producirse tal como se indica en la figura 1. La primera rotura corresponde a los alambres estirados en frío (diagonal) y la segunda a los templados. Estas diferencias provienen de la distinta textura de ambos tipos de acero.

Para evitar las roturas por corrosión bajo tensión hay que impedir, ante todo, cualquier ataque químico (gases corrosivos en la atmósfera) de los rollos durante el almacenaje. También debe cuidarse de que no se produzcan entalladuras durante la colocación de los hilos y, por supuesto, hay que asegurarse de que el hormigón protege adecuadamente a las armaduras.

Si, de todos modos, es de esperar que se produzca en la obra algún ataque químico-atmosférico o que no puedan evitarse las entalladuras, conviene seleccionar un tipo de acero poco susceptible a la corrosión bajo tensión.

Para tener alguna idea de dicha susceptibilidad puede servir el ensayo de Wyszomirski. Para ello, se colocan las muestras de alambre en un baño a $105^{\circ} \mathrm{C}$ y se someten a una cierta tensión. El tiempo requerido para la rotura de la probeta es un índice de la resistencia a la corrosión bajo tensión.

La composición del baño es:

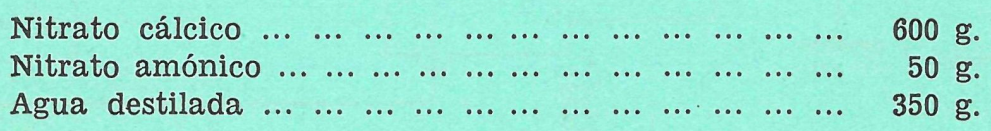

Mediante este ensayo se ha podido comprobar una gran diferencia de comportamiento entre algunos tipos de alambres. Por ejemplo, los aceros destensionados (ver I) se comportan mucho mejor que los templados y laminados. Para un tipo determinado de acero, no se encuentran diferencias cualquiera que sea su diámetro. 
Aunque los ensayos de resistencia a la corrosión bajo tensión no son, por ahora, concluyentes, como medida de precaución no deben utilizarse alambres templados cuando son de esperar condiciones agresivas en la obra (4). Rôs (7) indica que los alambres templados por inmersión y estirados en frío exhiben una elevada resistencia a la corrosión bajo tensión.

\section{Ensayo de uniformidad y ausencia de defectos}

Existe alguna posibilidad de control de uniformidad en los alambres por medio del aparato SD.1 (2). Este instrumento, al parecer, trabaja por inducción y, probablemente, está basado en medidas magnéticas de reluctancia por medio de un transformador diferencial.

Esta disposición es muy sensible a los cambios en el endurecimiento (por templado u otro tratamiento) de los aceros al carbono. Se comprende que será fácil diseñar un aparato para el ensayo continuo de los alambres, utilizando un esquema como el de la figura 2.

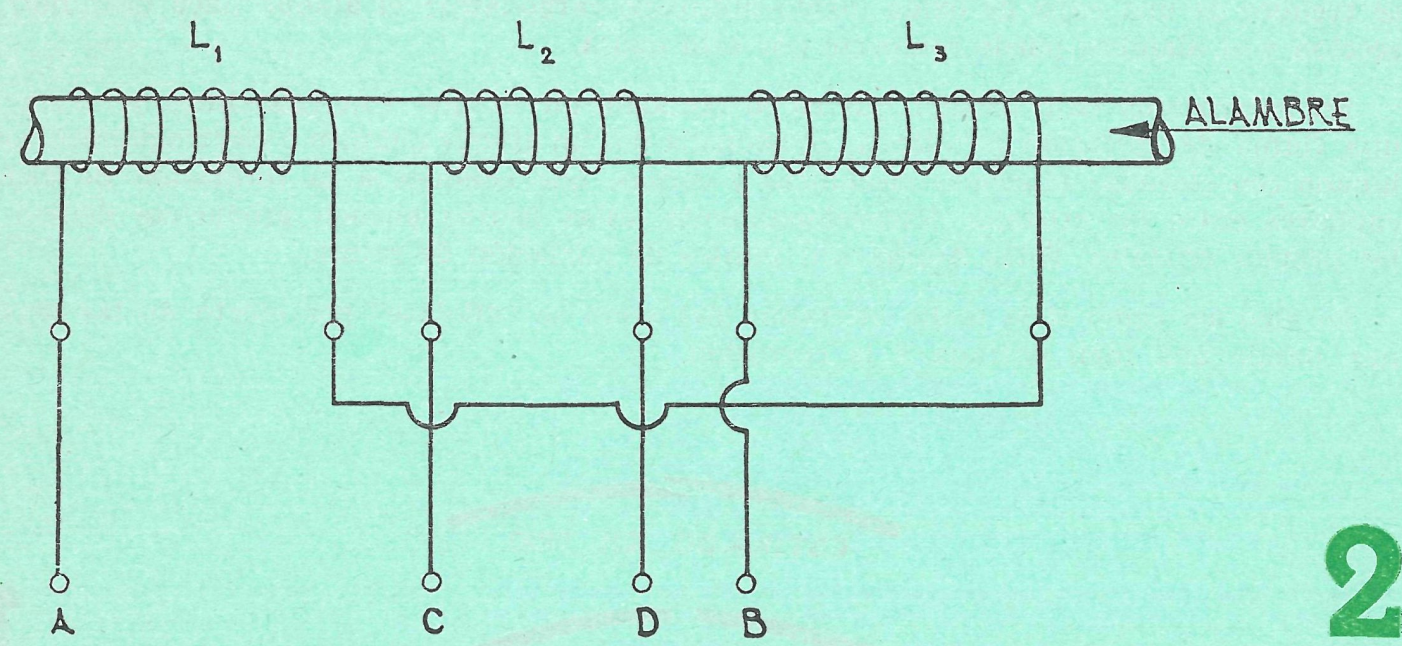

Se disponen tres bobinas iguales, $\mathrm{L}_{1}, \mathrm{~L}_{2}, \mathrm{~L}_{3}$, conectadas tal como se indica en la figura 2 , y se aplica una tensión alterna entre los extremos CD. Entre A y B no existirá tensión alguna si el acero que forma el núcleo de este transformador diferencial es de calidad homogénea.

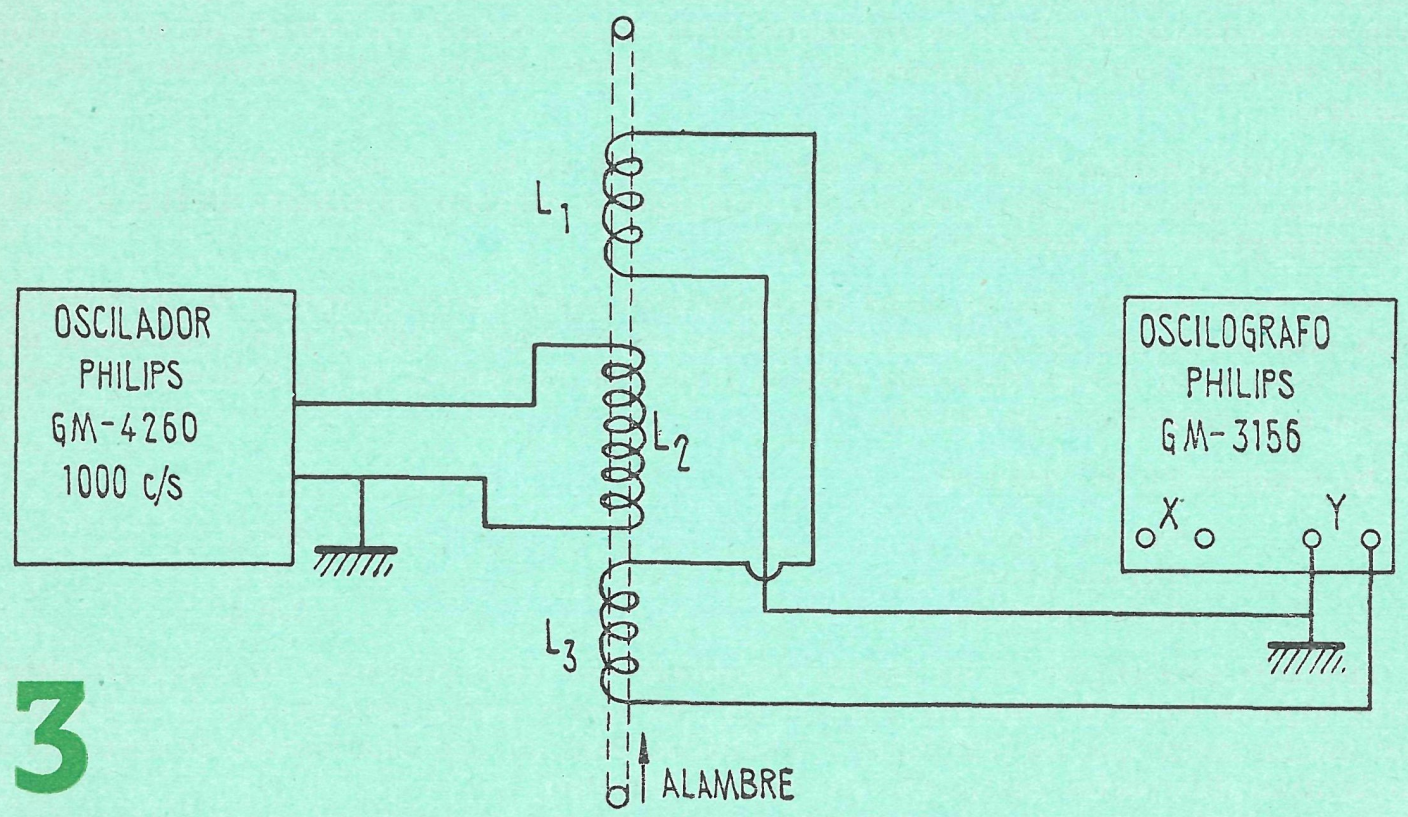


Pero si una mitad de la porción de alambre introducido en las bobinas presenta una reluctancia diferente de la otra mitad, el transformador se desequilibrará y aparecerá una tensión entre A y B.

Experimentalmente se ha comprobado (6) que puede operarse con una corriente alterna de $50 \mathrm{c} / \mathrm{s}$ (corriente de red), pero, en nuestros ensayos, hemos visto que puede aumentarse la sensibilidad de la medida trabajando a $1.000 \mathrm{c} / \mathrm{s}$. Teniendo esto en cuenta, y utilizando para la determinación de la tensión en $\mathrm{AB}$ un oscilógrafo de rayos catódicos, llegamos al montaje esquematizado en la figura 3.

La salida en AB se aplica a las placas verticales del GM.3156, mientras que la bobina $\mathrm{L}_{2}$ se alimenta con un oscilador a $1.000 \mathrm{c} / \mathrm{s}$ tipo GM.4260.

\section{Deralles constructivos}

La parte fundamental del aparato es el transformador diferencial (fig. 4). Se trata de un carrete de plexiglás con un orificio central de $9 \mathrm{~mm}$ de diámetro, provisto de tres acanaladuras que llevan otras tantas bobinas. El diámetro del orificio da el calibre máximo del alambre que puede medirse.

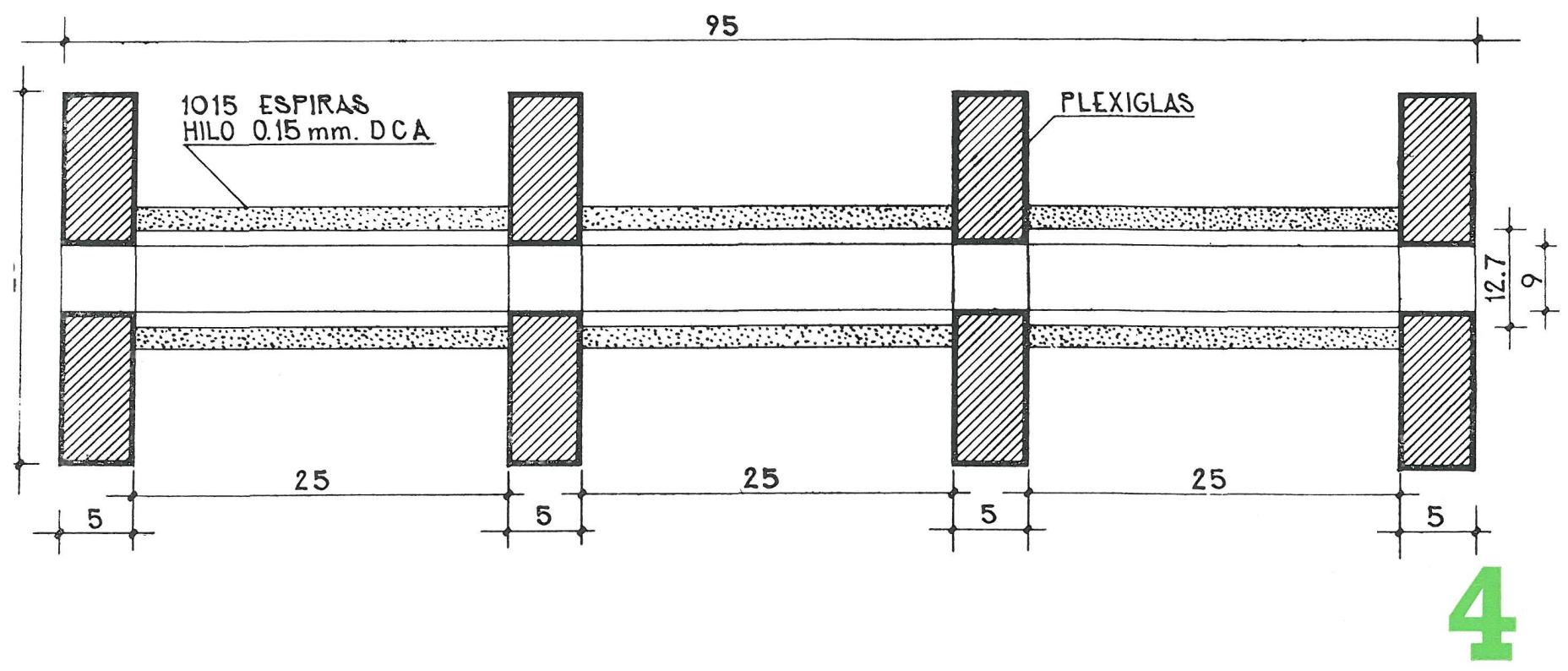

El transformador ya bobinado presenta el aspecto indicado en la figura 5. La figura 6 muestra el blindaje de latón que alberga al transformador, y en ella pueden verse las boquillas o racores, cuyo orificio interior ha de ser igual al diámetro del alambre que se ensaya. Hay un juego de boquillas para los diámetros de alambre más comunes.

La figura 7 corresponde al aparato terminado, y en ella pueden verse el oscilógrafo, el oscilador de $1.000 \mathrm{c} / \mathrm{s}$ y el transformador en su blindaje. Estos dos últimos montados sobre el oscilógrafo.

\section{Funcionamiento del aparato}

Una vez encendido y estabilizado el aparato se va pasando el alambre, en forma continua, a través del transformador a una velocidad aproximada de $20 \mathrm{~cm} /$ segundo.

Cualquier defecto estructural del acero se refleja en la traza del oscilógrafo con mayor o menor amplitud, según la profundidad o importancia del defecto. En unos ensayos previos se hicieron experiencias con alambre comercial continuo de acero de $5 \mathrm{~mm}$ de diámetro. En determinadas zonas del mismo se provocó un destemplado parcial por calentamiento con un mechero de laboratorio. 

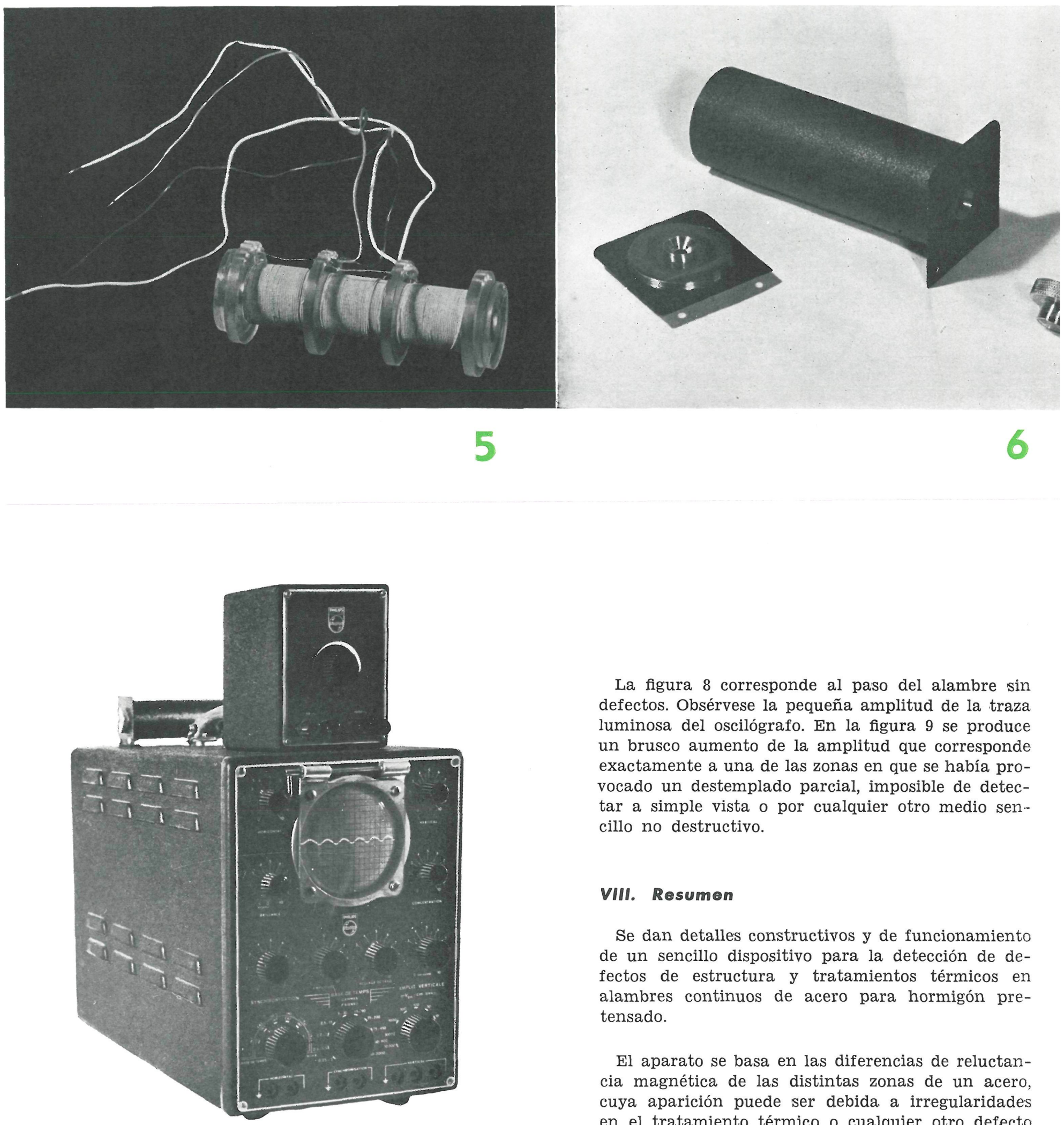

La figura 8 corresponde al parso del alambre sin defectos. Obsérvese la pequeña amplitud de la traza luminosa del oscilógrafo. En la figura 9 se produce un brusco aumento de la amplitud que corresponde exactamente a una de las zonas en que se había provocado un destemplado parcial, imposible de detectar a simple vista o por cualquier otro medio sencillo no destructivo.

\section{Resumen}

Se dan detalles constructivos y de funcionamiento de un sencillo dispositivo para la detección de defectos de estructura y tratamientos térmicos en alambres continuos de acero para hormigón pretensado.

El aparato se basa en las diferencias de reluctancia magnética de las distintas zonas de un acero, cuya aparición puede ser debida a irregularidades en el tratamiento térmico o cualquier otro defecto que dé lugar a una falta de homogeneidad en la calidad del alambre.

También se incluyen algunas consideraciones sobre la composición y características más comunes de los aceros para pretensado que son de uso general en la actualidad. 


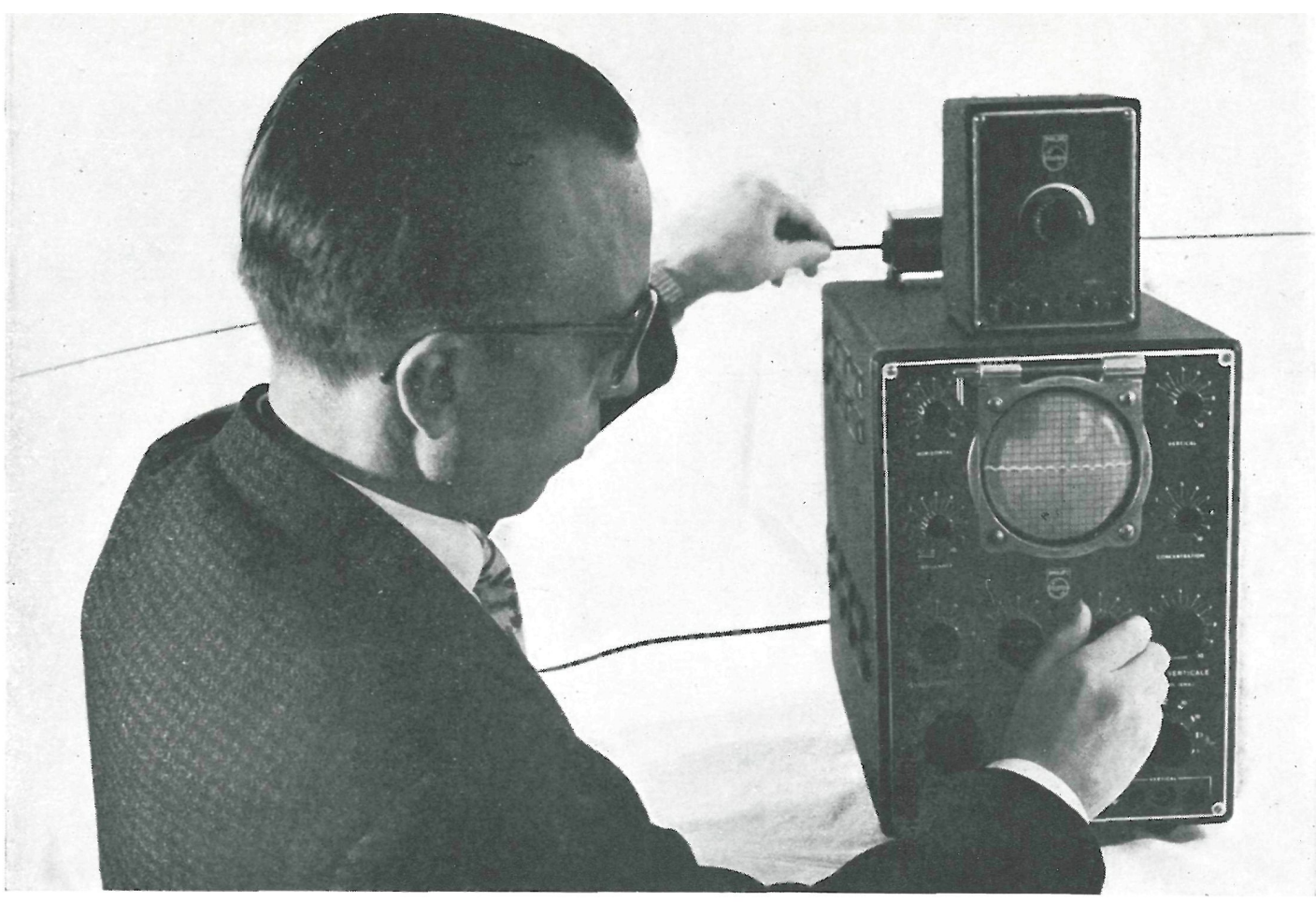

\section{Bibliografia}

(1) Millot, A.: Aceros para hormigón pretensado. II Congreso de la F. I. P., Amsterdam (1955).

(2) Schwier, F.: Alambres y barras de acero para hormigón pretensado. Symposium del R. I. L. E. M., IIa1, Lieja (1958).

(3) LEvi, F.: Resultados de una serie de experiencias sobre aceros para pretensado. II Congreso de la F. I. P., Amsterdam (1955)

(4) Bouvy, J. B.: Loc. cit.

(5) LeGat, A.: Aspectos metalúrgicos, metalográficos y económicos en la fabricación de aceros para pretensado. Symposium del R. I. L. E. M., IIa2, Lieja (1958).

(6) Förster, F.: Ensayo de la calidad sin destrucción por un procedimiento electromagnético. Technische Mitteilungen, cuaderno 3, 50 (1957).

(7) Roŝ, M. R.: El alambre de acero para pretensado considerado desde el punto de vista del fabricante y del proyectista. Symposium del R. I. L. E. M., IIb6, Lieja (1958).

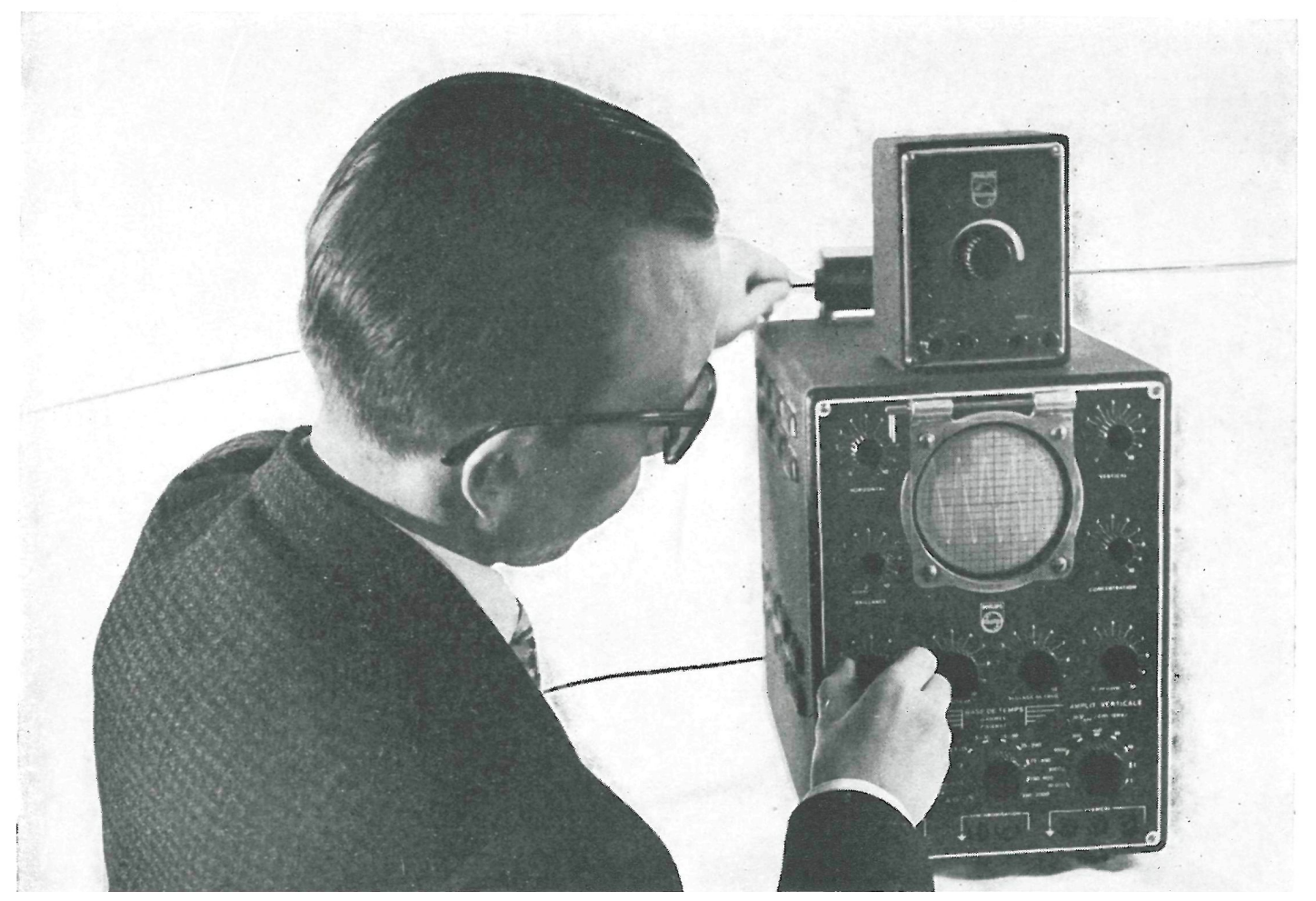

